\title{
Thyrotoxicosis Following Fine-Needle Aspiration Biopsy of A Thyroid Nodule: Case Report
}

John J. Orrego, M.D.

Department of Endocrinology and Metabolism, Colorado

Permanente Medical Group. 1375 East 20 ${ }^{\text {th }}$ Avenue, Denver CO

80205. Email: John.J.Orrego@kp.org

Dr John J. Orrego does not have any conflict of interest.

Fecha de recepción: 15/10/2016

Fecha de aceptación: 27/10/2016

\section{Abstract}

F ine-needle aspiration biopsy (FNAB) is a simple and generally safe office-based procedure that accurately and cost-effectively improves the diagnostic investigation of nonfunctioning thyroid nodules. Thyrotoxicosis following FNA has been rarely described in the medical literature. A case of post aspiration thyrotoxicosis is described here with the intent of increasing awareness of this likely underreported complication of FNAB.

Key works: Thyrotoxicosis= tirotoxicosis. Hyperthyroidism= hypertiroidismo. Fine-needle aspiration biopsy= biopsia tiroidea por aspiración. Thyroid nodule= nódulo tiroideo. Complication $=$ complicación

\section{Introduction}

Fine-needle aspiration biopsy (FNAB) is a common, simple, and generally safe office-based procedure that accurately and cost-effectively improves the diagnostic investigation of nonfunctioning thyroid nodules. Post-FNAB complications range from more frequently-occurring neck soreness and discomfort, local bruising, and vasovagal reactions, to less common recurrent laryngeal nerve palsy, needle track seeding of thyroid cancer, and post-aspiration thyrotoxicosis ${ }^{(1)}$. The latter was first reported in 1992, when its frequency was estimated to be close to $1 \%{ }^{(2)}$. Since that time, only one other case of post-aspiration thyrotoxicosis has been published in the English medical literature $^{(3)}$. In order to increase awareness of this, likely underreported, complication of FNAB, another case is presented.

\section{Case presentation}

A 78-year-old woman was referred to the Endocrinology Department for evaluation of a right thyroid nodule. She was clinically euthyroid and denied neck tenderness or pain, dysphonia, dyspnea, dysphagia, or chocking sensation. She had no previous history of thyroid disorders or radiation therapy and reported no family history of thyroid cancer. She had asthma, hypertension and osteopenia. Medications included beclomethasone dipropionate and albuterol inhalers, losartan and prazosin. Blood pressure was $134 / 82 \mathrm{mmHg}$, pulse was 64 heart beats per minute, and BMI was $27 \mathrm{~kg} / \mathrm{m} 2$. She appeared well and was in no acute distress. She had no proptosis or pretibial myxedema. Neck palpation demonstrated a large visible and palpable, mobile, smooth, non-tender right thyroid nodule. The trachea was slightly deviated to the left. The Pemberton's sign was negative. TSH was normal. Thyroid ultrasound demonstrated a 4.2 (L) × 2.2 (T) x 2.3-cm (AP) mostly solid, spongiform, moderately hypervascular nodule occupying most of the right thyroid lobe (figure 1). There were several subcentimeter thyroid cysts in the left thyroid lobe. Ultrasound-guided FNAB was undertaken in the office. Four passes were performed by twirling and probing 27 gauge needles attached to $5 \mathrm{~mL}$ syringes. There were no immediate post-aspiration complications. The specimen was moderately cellular with scattered groups of benign-appearing follicular epithelial cells, which were arranged in mono layers. Numerous macrophages were present along with amorphous debris. Colloid was present. No diagnostic features of malignancy were identified. These findings were consistent with a benign follicular (colloid) thyroid nodule.

Figure 1. Mostly solid spongiform thyroid nodule with some cystic degeneration and increased vascularity.

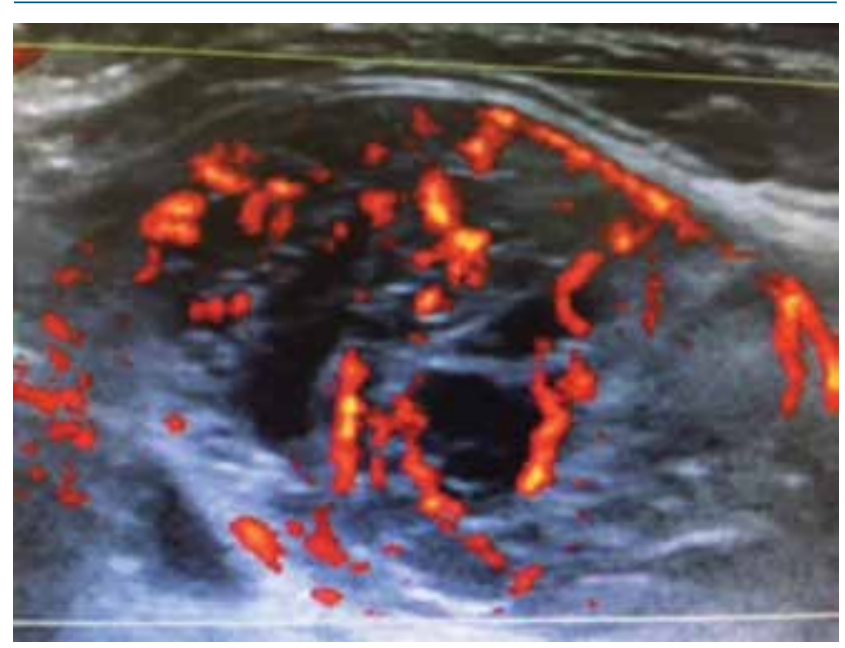


She noted mild neck tenderness and dysphagia after the FNAB, which got progressively worse. She reported palpitations 3 days later. She had worsening fatigue, anorexia, and weakness. Fifteen days after the FNAB, she presented to the emergency room with palpitations, dyspnea, and an 8-lb weight loss. She was found to have paroxysmal supraventricular tachycardia. Thyroid function tests demonstrated overt hyperthyroidism (table 1). Chest CT angiogram ruled out pulmonary embolism. Warfarin and Coreg were initiated.

She followed-up in the endocrine clinic 3 days after hospital discharge. She reported no fever, chills, compressive neck symptoms, or dyspnea. Her neck pain had resolved. BP was 156/81 $\mathrm{mm} \mathrm{Hg}$ and pulse was 62 heart beats per minute. Her neck was not tender and her right thyroid nodule appeared stable. Thyroid function tests showed persistent hyperthyroidism. Thyroid-stimulating immunoglobulin (TSI) and thyroid peroxidase antibody (TPO) titers were normal. Methimazole $20 \mathrm{mg}$ per day was begun on $9 / 16 / 15$, which was progressively tapered down because of elevated TSH concentrations (table 1) and finally discontinued on 2/22/16.

\section{Discussion}

A case of thyrotoxicosis following FNAB of a spongiform thyroid nodule with some cystic degeneration is reported. Kobayashi et $\mathrm{l}^{(2)}$ described 6 patients with thyroid cysts who developed thyrotoxicosis 2 to 20 days after cyst aspiration. All thyroid lesions were $\geq 3 \mathrm{~cm}$ in maximal diameter. The initial chief complaint was cyst recurrence and/or neck pain with no hyperthyroid symptoms in all but one patient. The 24-h radioiodine uptake was low in the 4 patients who underwent thyroid scan and uptake. The duration of the thyrotoxic phase was short ( $\leq 30$ days)

The only other published case of post aspiration thyrotoxicosis was reported in $2005^{(3)}$. A 39-year-old woman developed thyrotoxicosis as a result of acute suppurative thyroiditis due to Staphylococcus aureus following FNAB of a large left thyroid cyst. Due to abscess formation and refractoriness to antibiotic treatment, left thyroid lobectomy was undertaken a month later, with resolution of her symptoms.

The patient reported here was diagnosed with overt hyperthyroidism 15 days after FNAB of a 4.2-cm spongiform thyroid nodule with some cystic degeneration. Thyroid scan and uptake were not performed since intravenous iodinated contrast was administered upon hospital admission. Because of the severity of her hyperthyroid symptoms, methimazole was initiated with resolution of the hyperthyroid state within 3 weeks.

The etiologic mechanism of hyperthyroidism after FNAB is unclear. Transient thyroid inflammation with leakage of thyroid hormones into the cystic lesion and subsequent transfer into the circulation has been proposed ${ }^{(2)}$. Given that other etiologies of hyperthyroidism on the patient reported here were unlikely,
Table 1.

\begin{tabular}{l|c|c|c}
\hline \multicolumn{1}{|c|}{ Date } & $\begin{array}{c}\text { TSH (0.32-5.5 } \\
\mathbf{m l U} / \mathbf{m L})\end{array}$ & $\begin{array}{c}\text { FT4 (0.7-1.8 } \\
\mathbf{n g} / \mathbf{d L})\end{array}$ & $\begin{array}{c}\text { FT3 (2.4-4.2 } \\
\mathbf{n g} / \mathbf{d L})\end{array}$ \\
\hline $7 / 31 / 15$ & 2.31 & & \\
\hline $9 / 11 / 15$ & 0.008 & 3.14 & 6.25 \\
\hline $9 / 14 / 15$ & 0.017 & 3.0 & 4.75 \\
\hline $10 / 5 / 15$ & & 1.1 & 2.48 \\
\hline $11 / 16 / 15$ & 7.78 & & \\
\hline $12 / 16 / 15$ & 12.71 & 0.9 & \\
\hline $1 / 15 / 16$ & 7.36 & & \\
\hline $2 / 18 / 16$ & 4.63 & & \\
\hline $4 / 6 / 16$ & 2.76 & & \\
\hline
\end{tabular}

this explanation seems to hold true. The nodule of the current patient is no autonomous since her TSH levels had been in the 2.3-4.8 $\mathrm{mIU} / \mathrm{mL}$ range over the past 10 years. The short duration of the thyrotoxic phase and the normal TSI level make Graves' disease very unlikely. The absence of thyroid heterogeneity by ultrasound and the undetectable TPO antibody rule out Hashitoxicosis. Although she had thyroidal pain lasting for about 2 weeks, this was precipitated by the FNAB and not by a viral infection, as seen in DeQuervain's thyroiditis. Painless thyroiditis, which is usually associated with positive anti-thyroid antibodies, does not cause thyroidal pain. She had never been on amiodarone and was taken no iodine supplements. Administration of iodinated IV contrast occurred after she was already hyperthyroid. Clinical history, physical exam, and spontaneous improvement are against acute suppurative thyroiditis.

Similar to previous cases, her thyroid nodule was large, she had post aspiration thyroidal pain, and the hyperthyroid state was diagnosed soon after the FNAB and lasted less than a month. The methimazole most likely caused her subsequent subclinical hypothyroid state by inhibiting the synthesis of new thyroid hormone, without affecting the release of the already stored contents. Contrary to those other cases, this patient's nodule was mostly solid rather than cystic and she had overt hyperthyroidism. Markers of inflammation such as sedimentation rate and $\mathrm{C}$ reactive protein and thyroid hormone levels in the cystic fluid were not obtained.

Destructive thyroiditis is postulated as the mechanism of post aspiration thyrotoxicosis. Repetitive trauma to the thyroid nodule with subsequent extracellular leakage of thyroid hormones and their systemic absorption via increased local vascularity may be the culprit of this likely underreported complication of FNAB.

\section{References}

1. Polyzos SA and Anastasilakis AD. Clinical complications following thyroid fine-needle biopsy: a systematic review. Clin Endocrinol. 2009; 71:157-165.

2. Kobayashi A, Kuma K, Matsuzuka F, Hirai K, Fukata S, Sugawara M. Thyrotoxicosis after needle aspiration of thyroid cyst. J Clin Endo Metab. 1992; 75: 21-24.

3. Nishihara E, Miyauchi A, Matsuzuka F, Sasaki I, Ohye H, Kubota S, et al. Acute suppurative thyroiditis after fine-needle aspiration causing thyrotoxicosis. Thyroid. 2005; 15: 1183-1187. 